\title{
Determination of Radiotherapy-Related Acute Side Effects; A Starting Point for the Possible Implementation of a Clinical Pharmacy Services in the Radiological Unit in Turkey
}

\author{
Anmar Al-Taie ${ }^{1, *}$, Aygül Köseoğlu² \\ 'Department of Pharmacy, Asoul Aldean University College, Baghdad, IRAQ. \\ 2Oncology Center, Dr. Lütfi Kırdar Kartal Teaching and Research Hospital, Istanbul, TURKEY.
}

\begin{abstract}
Background: Although it is considered as important part in cancer therapy, radiotherapy is associated with many acute side effects. The clinical pharmacist in clinical oncology practice provides a well-defined role in the assessment and monitoring of side effects during treatment. The aim of this study was to explore Radiotherapy-Related Acute Side Effects (RRASEs) in a real-life setting at the radiology unit and whether there is a possibility for providing radiology unit-based clinical pharmacy supportive services in collaboration with other healthcare professionals for cancer patients admitted for radiotherapy application. Methods: A retrospective descriptive study was carried out on cancer patients admitted for radiotherapy application at the radiology unit. During the study, detection of RRASEs incidence and their outlined treatments were assessed. Results: RRASEs observed was 494 with a mean number of $6.175 \pm 2.88$ per patient. A high incidence of RRASEs was encountered among male patients $61.3 \%$. Nausea and vomiting were the most common acute side effects encountered $82.5 \%$ followed by loss of appetite $73.8 \%$. There was a significant correlation between the incidence of RRASEs and a number of factors that were related to age
\end{abstract}

greater than 60 years $(P=0.0003)$, cigarette smoking $(P=0.0084)$, comorbidities $(P=0.0328)$, carcinoma type and stage $(P=0.0098)$ and $(P=0.0001)$ respectively. Conclusion: Radiotherapy application is associated with the incidence of different acute side effects that require proper treatment and follow-up. This could be further achieved through the introduction of the clinical pharmacist services in collaboration with other healthcare professionals for better patient-related health outcomes.

Key words: Acute Side Effects, Cancer, Clinical Pharmacy Services, Radiology Unit, Turkey.

\section{Correspondence}

Dr. Anmar Al-Taie

Department of Pharmacy, Asoul Aldean University College, Baghdad, IRAQ.

Phone: +964 7727369380

Email: altaii1978@gmail.com

DOI: 10.5530/jyp.2019.11.89

\section{INTRODUCTION}

Radiotherapy is an integral part of cancer therapy and nowadays it is considered as an important choice of treatment for the majority of cancer patients. ${ }^{1}$ Two types of radiotherapy-related side effects are documented and they are classified as early acute and late chronic. The early acute side effects are usually seen during radiotherapy application or just after its completion, which end up within four to six weeks. The late side effects are considered as chronic or permanent and foreseen within months to years after radiotherapy completion. ${ }^{2}$ Several oncology literatures reported that radiotherapy application is associated with numerous acute side effects including acute skin reactions (90\%); gastrointestinal toxicity (40 to $80 \%$ ) affecting oral mucosa, larynx and salivary function; and genitourinary toxicity (40 to $74.5 \%$ ) affecting prostate and cervical tissues. $^{3-6}$

Healthcare professionals should have a profound attention to minimize the incidence of both complications during cancer therapy. ${ }^{7}$ The proper overcome and management of these side effects could be accomplished through provision of Clinical Pharmacy Services (CPSs). One of the main objectives of the clinical pharmacist in oncology practice is the assessment and monitoring of side effects revealed by patients during treatment. ${ }^{8}$ Earlier studies indicate that patient education can reduce treatment complications, improve physical and psychosocial outcomes, influence patients' desire to complete the course of therapy, minimize unnecessary hospital admissions and improve patients' quality of life. ${ }^{9-11}$ Despite these facts, therapy-related complications still not considered as a relevant problem by a large proportion of healthcare professionals and the clinical pharmacist is still an under-utilised resource. ${ }^{12,13}$

The purpose of this study was to explore in a real-life setting the incidence of RRASEs at a radiology unit and whether there is a possibility for providing radiology unit-based clinical pharmacy supportive services to ease the reduction and management of these complications in collaboration with other healthcare professionals for cancer patients admitted at the radiology unit in one of the main hospitals at the Anatolian part of Istanbul-Turkey.

\section{MATERIALS AND METHODS}

The study was designed as a retrospective descriptive; single-centre analysis. Patients were screened from January through July 2015. Approval of this study was granted from the Ethical Committee of Dr. Lütfi Kırdar Kartal Teaching and Research Hospital. The research was conducted at the radiology unit of Dr. Lütfi Kirdar Kartal Teaching and Research Hospital as one of the principle hospitals in the Anatolian part of Istanbul, Turkey.

Irrespective of the type of carcinoma, all adult patients over the age of 18 years old, newly diagnosed with cancer and referred for receiving radiotherapy, were enrolled in this study and were checked for the incidence of RRASEs. A number of patients receiving radiotherapy at the radiology unit during the above period were used to estimate the sample size. In 
order to remove the effect of earlier treatment, patients treated with either a previous chemotherapy or radiotherapy were undertaken a washout period of 12 months. Exclusion criteria included patients with neoadjuvant chemotherapy, those receiving chemotherapy concomitantly, patients received either chemotherapy or radiotherapy at a period of less than 12 months and patients with incomplete data. The details of the patients were screened taking into account patients' socio-demographic data (Age, gender, cigarette smoking, presence of comorbid conditions), disease characteristics (Previous chemotherapy, previous radiotherapy, radiation-applied area, type and stage of diagnosed cancer). The data were collected from the medical records of the patients and used to structure a detailed health report for the patients.

\section{Outcomes and analysis}

In the radiotherapy unit, radiation oncologists and nursing staff are the main healthcare team providing therapy services. CPSs are usually lacking as there is no clinical pharmacist presented to provide clinical pharmacy care services. In the outpatient radiology unit of our hospital, the observed RRASEs are usually recorded in the medical charts of the patients who received radiotherapy. These reported acute side effects were reviewed and evaluated by the researcher clinical pharmacist in order to assess the incidence of RRASEs and considered as the primary objective of this study. The RRASEs were assessed and recorded based upon those described by the American Cancer Society. ${ }^{14}$ Proper management of cancer patients suffering from these complications was also reported and considered as the secondary objective of this study.

The SPSS version 16 was used for statistical analysis. Descriptive analysis was used to describe the study population and the results were expressed in numbers, percentages, mean values and standard deviations. Comparison of mean between any two groups was carried out using independent sample $t$-test. Association between categorical variables was assessed using either chi-square or corrected chi-square test. Chi-square test was used to compare between percentages in this study. The level of significance used for the statistical analysis was considered as $P<0.05$.

\section{RESULTS}

Table 1 presents the socio-demographic characteristics of the recruited study participants who met the study's inclusion criteria. As 16 patients met the exclusion criteria, 80 patients were enrolled in this study. The mean age of the patients was $58.4 \pm 14.8$ years ranging from 60 to 92 years; most of them were males $(55,68.8 \%)$. The majority of the patients were diagnosed with stage IV cancer $(67,83.8 \%)$ with no previous history of radiotherapy $(77,96.2 \%)$. Lung cancer was the most common type (28, $35 \%)$ and thorax represented the most common radiation-applied area $(36,45 \%)$.

The total number of RRASEs observed was 494 with mean number of $6.175 \pm 2.88$ per patient. 'Males and females' incidence of RRASEs was $303(61.3 \%)$ and 191(38.7\%), ( $P=0.0001)$ respectively. Figure 1 shows the incidence of RRASEs among study population. The findings demonstrated that Radiotherapy-Induced Nausea and Vomiting (RINV) was the most common acute side effects encountered $(66,82.5 \%)$ followed by loss of appetite $(59,73.8 \%)$; skin reactions $(51,63.8 \%)$. Radiotherapy-related oral complications were also of high incidence among patients of this study. These include stomatitis $(55,68.8 \%)$; swallowing difficulties $(45,56.2 \%)$; mouth ulcers and dry moth $(43,53.8 \%)$ and metallic taste (40, 50\%). Other RRASEs are shown in Figure 1.

Table 2 shows whether there is a possible correlation between characteristics of the study population and the incidence of RRASEs. Patients greater than 60 years old demonstrated a significant higher incidence of dry mouth $(P=0.0437)$, metallic taste $(P=0.0003)$. Patients diagnosed with stage IV cancer were significantly suffering from RINV $(n=46$,
Table 1: Demographic and Clinical Characteristics of Study Population.

\begin{tabular}{|c|c|c|}
\hline Variable & $\begin{array}{l}\text { No. of Patients } \\
\qquad(n=80)\end{array}$ & $\begin{array}{c}\text { Percentage } \\
\text { (\%) }\end{array}$ \\
\hline $\begin{array}{c}\text { Age (years), Mean } \pm \text { SD } \\
\text { (Range) }\end{array}$ & $58.4 \pm 14.8,60$ to 92 & -------- \\
\hline \multicolumn{3}{|l|}{ Gender } \\
\hline Males & 55 & 68.8 \\
\hline Females & 25 & 31.2 \\
\hline \multicolumn{3}{|l|}{ Cigarette Smoking } \\
\hline Yes & 25 & 31.2 \\
\hline No & 55 & 68.8 \\
\hline \multicolumn{3}{|l|}{ Comorbid Conditions } \\
\hline Yes & 20 & 25 \\
\hline No & 60 & 75 \\
\hline \multicolumn{3}{|l|}{ Cancer Stage } \\
\hline Stage III & 13 & 16.2 \\
\hline Stage IV & 67 & 83.8 \\
\hline \multicolumn{3}{|l|}{ Previous Chemotherapy } \\
\hline Yes & 42 & 52.2 \\
\hline No & 38 & 47.5 \\
\hline \multicolumn{3}{|l|}{ Previous Radiotherapy } \\
\hline Yes & 3 & 3.8 \\
\hline No & 77 & 96.2 \\
\hline \multicolumn{3}{|l|}{ Radiation-Applied Area } \\
\hline Thorax & 36 & 45 \\
\hline Head & 25 & 31.2 \\
\hline Pelvis & 15 & 18.8 \\
\hline Vertebra & 4 & 5 \\
\hline \multicolumn{3}{|l|}{ Type of Carcinoma (CA) } \\
\hline Lung CA & 28 & 35 \\
\hline Breast CA & 17 & 21.2 \\
\hline Brain CA & 12 & 15 \\
\hline Skin CA & 12 & 15 \\
\hline Colorectal CA & 4 & 5 \\
\hline Prostate CA & 4 & 5 \\
\hline Pelvis CA & 3 & 3.8 \\
\hline $\begin{array}{l}\text { Total number of RRASEs, } \\
\text { Mean } \pm \text { SD }\end{array}$ & $494,6.175 \pm 2.88$ & \\
\hline Males incidence (n, \%) & $303(61.3)$ & -------- \\
\hline Females incidence (n, \%) & $191(38.7)$ & \\
\hline
\end{tabular}

$P=0.0001)$; skin reactions $(n=37, P=0.0001)$ and stomatitis $(n=44$, $P=0.0001$ ). It was found that cigarette smoker patients were also significantly at higher risk for developing different oral-related complications $(P=0.0084)$, including both mouth ulcers and dry mouth $(n=12)$, metallic taste $(n=22)$; stomatitis $(n=20)$, swallowing difficulties $(n=21)$. In addition, patients suffering from comorbid conditions also experienced significant incidence of RRASEs $(P=0.0328)$, Table 2 .

Regarding the type of cancer involved, there was a significant correlation between the cancer type and the incidence of RRASEs particularly RINV, skin reactions and different oral complications, including lung cancer $(P=0.0098)$, breast cancer $(P=0.0009)$ and skin cancer $(P=0.0287)$. For the radiation-applied area (Thorax vs. head), it was found that patients 
Table 2: Stratification of RRASEs Incidence by the Patients Demographic and Clinical Characteristics.

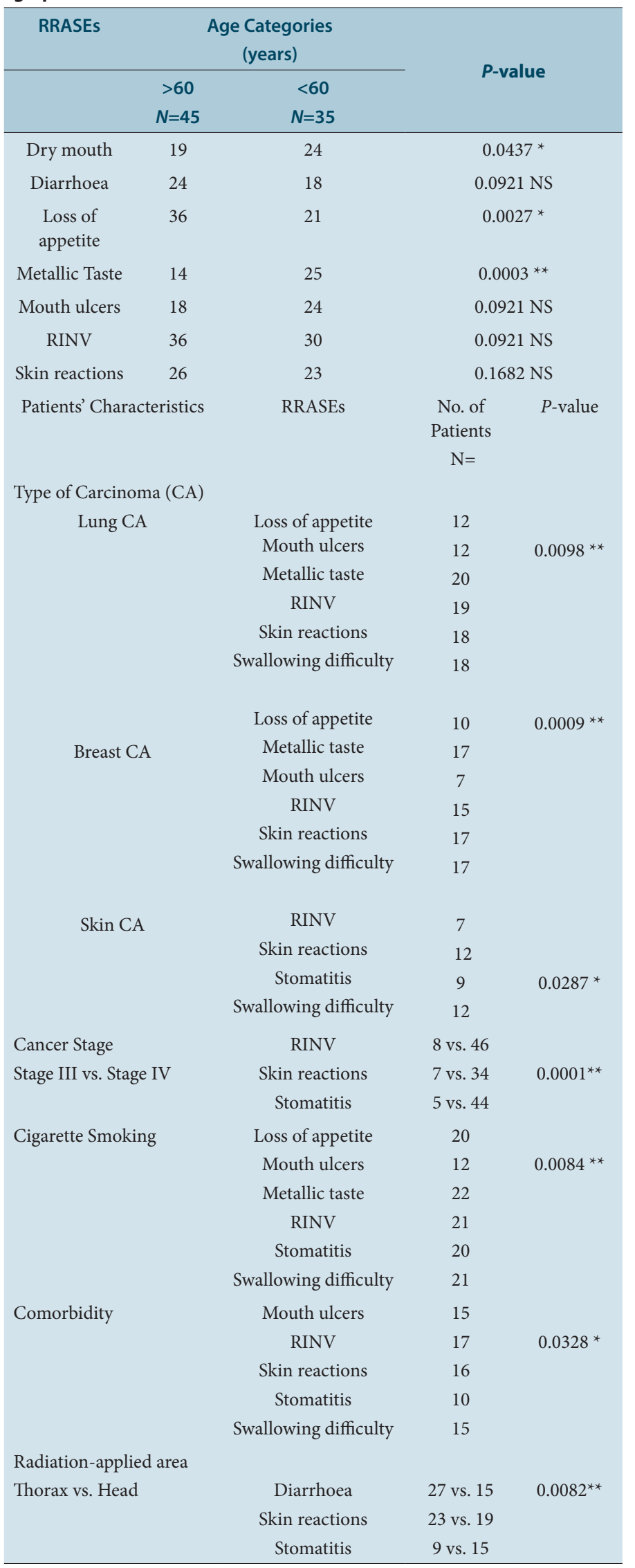

${ }^{\star}(P<0.05),{ }^{* *}(P<0.01)$, NS: Non-Significant.
Table 3: RRASEs Treatment Recommended for Part of the Study Population.

\begin{tabular}{cccc}
\hline RRASEs & $\begin{array}{c}\text { Suffering Patients } \\
(\text { Total=80) } \\
\mathbf{N}(\%)\end{array}$ & $\begin{array}{c}\text { Treated } \\
\text { Patients } \\
\text { N, }(\%)\end{array}$ & P-value \\
\hline Diarrhoea & $42(52.5)$ & $10(23.8)$ & $0.0003^{* *}$ \\
Loss of appetite & $59(73.8)$ & $12(20.3)$ & $0.0001^{* *}$ \\
Metallic taste & $40(50)$ & $2(5)$ & $0.0001^{* *}$ \\
RINV & $66(82.5)$ & $23(34.8)$ & $0.0001^{* *}$ \\
Skin reactions & $51(63.8)$ & $41(80.4)$ & $0.0086^{* *}$ \\
Stomatitis & $55(68.8)$ & $32(58.1)$ & $0.0423^{*}$ \\
\hline
\end{tabular}

${ }^{*}(P<0.05),{ }^{* *}(P<0.01)$.

receiving radiotherapy applied to the thorax area was associated with significant incidence of diarrhoea and skin reactions $(P=0.0082)$.

Treatment of some of the detected RRASEs is presented in Table 3. It was found that $23.8 \%(P=0.0003)$ and $34.8 \%(P=0.0001)$ of the patients had a treatment for diarrhoea and for nausea and vomiting respectively. $20.3 \%$ ( $P=0.0001)$ of the patients were treated for loss of appetite. For radiotherapy-related oral complications including metallic taste, only $5 \%(P=0.0001)$ were subjected to receive treatment, while for stomatitis $58.1 \%(P=0.0423)$ of the patients were received appropriate treatment. For skin reactions $80.4 \%(P=0.0806)$ of the patients were recommended treatment. Figure 2 also represented that not all patients complained RRASEs were prone to treatment.

\section{DISCUSSION}

In cancer therapy, radiotherapy provides good clinical and therapeutic outcomes, however these beneficial results are associated with the incidence of many side effects and severe complications. ${ }^{6,15}$ The findings of this study revealed the high incidence of RRASEs (Table 1). It is well known that the incidence of side effects including RRASEs could be related to a number of factors, such as advance age of the patients, gender, presence of comorbid conditions, advanced cancer stage (III or IV), cancer type, dose of radiation applied, social habits, fatigue and psychological aspects. ${ }^{16,17}$ Earlier studies were consistent with the findings of our study which reported high and frequent acute complications from radiotherapy application that manifest during or after the completion of therapy. ${ }^{3}$ Vaz et al. ${ }^{4}$ found that the incidence of acute radiotherapy toxicity was as high as $93.5 \%$ among gynaecological cancer patients undergoing pelvic radiotherapy. Andreyev $\mathrm{J}^{18}$ also reported that almost all patients experience gastrointestinal toxic symptoms following pelvic or abdominal radiotherapy.

RINV was the first most common and troublesome acute side effect encountered $(82.5 \%)$. It is postulated that radiotherapy particles causes activation of the central emetogenic centres in the brain, which leads to nausea and emesis. ${ }^{19}$ Results of this study are in agreement with Feyer $\mathrm{P}$ et al. study. ${ }^{19}$ which found that $50 \%$ to $80 \%$ of cancer patients undergoing radiotherapy may show nausea and vomiting during and/or after treatment. The high incidence of RINV is multifactorial ${ }^{20}$ which might be related to radiation treatment itself (Administered dose, applied area), patient-related factors, including gender (Males incidence $61.3 \%$, $P=0.0001)$, age and health status, such as cigarette smoking $(P=0.0084)$ and comorbidities $(P=0.0328)$, psychological state, tumour stage (stage IV, $P=0.0001$ ) and cancer type (such as lung cancer, $P=0.0098$ ) as presented in Table 2. Furthermore, these complications may cause interruption of therapy, negatively affect patients' healing and recovery process, 


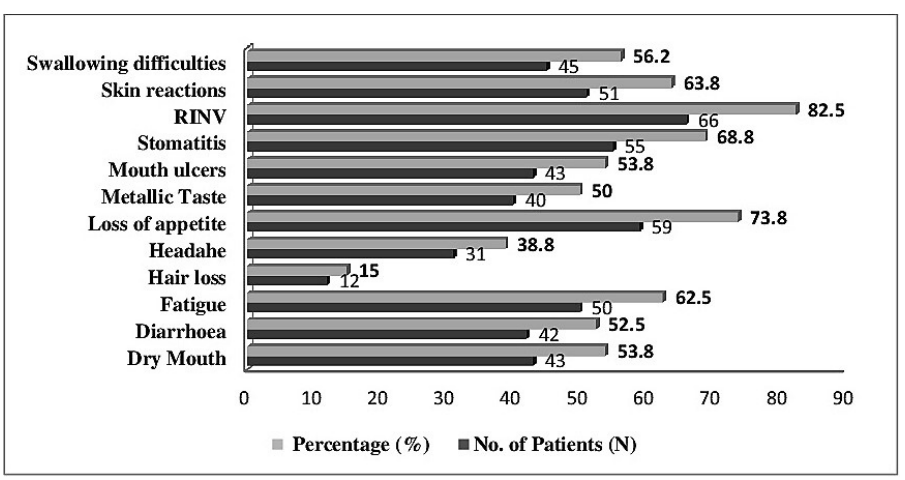

Figure 1: Incidence of RRASEs among Study Population.

response to treatment, sense of well-being, quality of life and increase the risk of infections. Therefore, early nutritional intervention is important to reduce dehydration, malnutrition, electrolyte imbalance, risk of infections, treatment toxicities and enhanced overall survival. ${ }^{20,21}$

The second most common observed RRASEs was loss of appetite (73.8\%). Radiotherapy frequently causes or exacerbates poor nutritional status among cancer patients that is related to the multiple experienced acute side effects encountered during this study, such as RINV, fatigue, diarrhoea and oral complications (Stomatitis, swallowing difficulties, mouth ulcers and metallic taste) alongside other related factors such as cancer type (Lung cancer, $P=0.0098$; breast cancer, $P=0.0009$ ) (Table 2). Meanwhile, malnutrition has been found to negatively affect patients' response to therapy and survival. It also associated with prolonged hospital stays, slower healing and recovery rates, increased complications and higher healthcare costs and mortality rates. ${ }^{22-24} \mathrm{~A}$ study by Guo Li et $a l .{ }^{25}$ reported that RRASEs had significant impact on weight loss during radiotherapy. Another study by Odelli C. et al. ${ }^{26}$ found that early and regular nutrition assessment and intervention results in improved treatment tolerance for oesophageal cancer patients receiving chemoradiation.

Radiotherapy-related oral complications were also among the most common and significant acute side effects observed in this study. These complications were manifested as metallic taste, mouth ulcers, stomatitis and swallowing difficulties. These findings were consistent with previous literature data which reported that radiotherapy-related oral complications are some of the most significant and unavoidable toxicities associated with cancer treatment ${ }^{27}$ and extensively reported that $90 \%$ to $100 \%$ of cancer patients receiving radiation therapy are suffering from different oral complications. ${ }^{28}$ These debilitating complications could severely affect mucosal surfaces for extended duration (3-12 weeks) than chemotherapy-related oral complications (3-12 days) and furthermore, could occur more aggressively in relation to certain risk factors, including advanced patient's age (Greater than 60 years old), gender (Males incidence $61.3 \%, P=0.0001)$, genetic predisposition, oral hygiene, cigarette smoking $(P=0.0084)$, alcohol drinking, presence of comorbidities $(P=0.0328)$, radiotherapy applied area (thorax, $P=0.0082$ ) and rate and dose of radiotherapy. ${ }^{29,30}$ as shown in Table 2. Eifel PJ et al. ${ }^{31}$ reported that the influence of serious radiotherapy complications was strongly correlated with certain patient-risk factors. Therefore, the reasons behind showing such correlation were to assess which patients' groups were subjected more to RRASEs incidence and to determine the type of acute complications commonly observed among specific study population. This could pay more attention at those populations to aid implementation of proper CPSs and to adjust and intensify these services for those highly risk patients.

Radiotherapy-related skin disorders are another common issue observed in this study with an incidence reaching $63.8 \%$. Even with advances in technology and changes to therapeutic regimens to lessen the burden of radiotherapy-related skin reactions, they remain as significant complica-

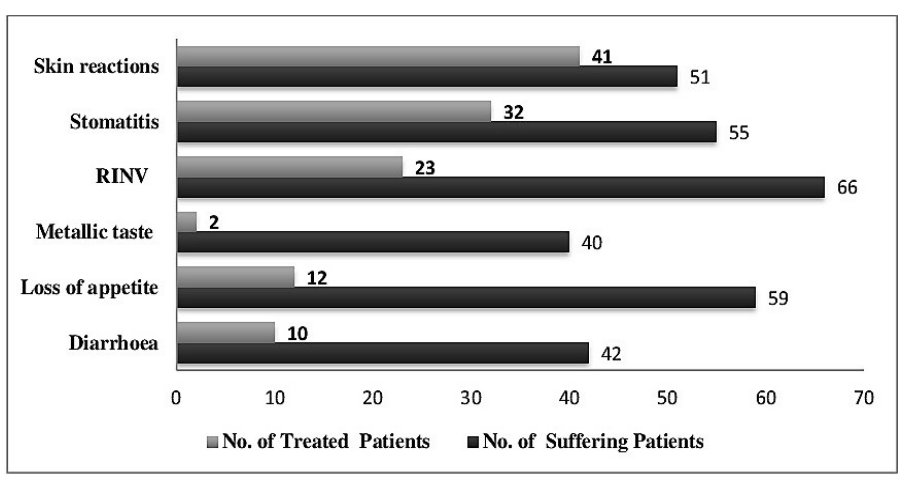

Figure 2: Differences between Patients Suffering- and Treated from RRASEs..

tions with an incidence up to $90 \% .6,32$ This can lead to premature interruption of therapy and potentially negatively influence cancer patients' control and cancer prognosis. Radiotherapy-related acute skin reactions observed in this study usually started within 1-4 weeks following therapy and manifested as mild red rash, itchy and peeling skin.

In this study, we observed that only a limited number of patients suffering from these acute complications were subjected to receive treatment as shown in Table 3 and Figure 2. RINV were the most common RRASEs observed, however we found that only $23(34.8 \%)$ out of 66 patients were treated. Only 10 patients (23.8\%) out of 42 had a treatment for diarrhoea. $12(20.3 \%)$ patients out of 59 were treated for loss of appetite. Regarding radiotherapy- related oral complications, only 2 (5\%) out of 40 patients and $32(58.1 \%)$ out of 55 patients were gotten a suitable treatment for metallic taste and stomatitis respectively. Furthermore, we observed a large number of RRASEs (494) among a small sample size number of study participants $(n=80)$. In this regard, those patients require more appropriate and extensive follow-up and further instructions in order to reduce and manage these complications during radiotherapy schedules. In oncology setting, clinical pharmacists are crucial team members and are adequately qualified to provide drug-related and consultative interventions. These interventions include patient education and counselling, boosting medication knowledge and side effects management. The interventions helped the patients to learn the expected therapy-related complications that could occurred during subsequent treatment cycles. ${ }^{33,34}$ Additionally, patients have to get a full knowledge about any supportive care medications, the reasons they are being used and self-monitoring of therapy-related side effects (When to expect the occurrence of these complications and how to manage them effectively). The lack of this knowledge could lead to increased hospital admissions, increased morbidity and reduced patients' quality of life. ${ }^{35-37}$ Hence, radiology unitbased clinical pharmacist role is essential in reviewing treatment plan, evaluating, minimising and getting more follow- up of side effects and in detecting other Drug-Related Problems (DRPs) to improve patient-related health outcomes. Therefore, for better implementation of CPSs, it is important to enhance the awareness and recognition of other healthcare professionals about the clinical pharmacist concerns.

\section{Strengths and limitations}

Many literatures have been discussed the incidence of radiotherapy-related complications, but to the best of our knowledge, this is the first study in Istanbul-Turkey which conducted the determination of RRASEs in a real-life setting in order to introduce CPSs and the importance of clinical pharmacist role at the radiology unit. This could be a starting point for developing prospective studies to explore the influence of CPSs and other therapy-related problems and thus building the gap between multidisciplinary team for better collaborative working at the radiology units of other hospitals. However, the study has some limitations that should be taken into consideration resulting from hidden confounders 
and biases due to the retrospective nature of the study. Another limitation included a small sample size of participants as the recruitment in this study included only patients meeting the eligible inclusion criteria. Finally, limitations included also patients' recruitment at the radiology unit of a single hospital and the researcher clinical pharmacist could not identify adherence for medications being recommended for patients which might limit the ability to detect improvements in some outcomes.

\section{CONCLUSION}

The results of this study pay more attention to help increase awareness about RRASEs and the pivotal role of the clinical pharmacist within the multidisciplinary team. During radiotherapy application, as patients experience many different acute side effects, the introduction of CPSs and radiology unit-based clinical pharmacist in collaboration with other healthcare professionals is considered elemental to meet the needs for better optimizing of treatment outcomes and improving patient satisfaction.

\section{ACKNOWLEDGEMENT}

The authors would like to express our gratitude to Dr. Atin AKSU and Dr. Athmar Dhahir for support and valuable suggestions during this study.

\section{CONFLICT OF INTEREST}

The authors declare no conflict of interest.

\section{ABBREVIATIONS}

CPSs: Clinical Pharmacy Services; RINV: Radiotherapy-Induced Nausea and Vomiting; RRASEs: Radiotherapy-Related Acute Side Effects.

\section{REFERENCES}

1. Delaney G, Jacob S, Featherstone C, Barton M. The role of radiotherapy in cancer treatment: Estimating optimal utilization from a review of evidence-based clinical guidelines. Cancer. 2005;104(6):1129-37.

2. Morris KA, Haboubi NY. Pelvic radiation therapy: Between delight and disaster. World J Gastrointest Surg. 2015;7(11):279-88.

3. Rosales AC, Esteves SC, Jorge J, Almeida OP, Lopes MA. Dental needs in Brazilian patients subjected to head and neck radiotherapy. Braz Dent J. 2009;20(1):74-7.

4. Vaz AF, Pinto-Neto AM, Conde DM, Costa-Paiva L, Morais SS, Esteves SB. Quality of life and acute toxicity of radiotherapy in women with gynecologic cancer: a prospective longitudinal study. Arch Gynecol Obstet. 2008;278(3):215-23.

5. Tan LT, Russell S, Burgess L. Acute toxicity of chemoradiotherapy for cervical cancer: The Addenbrooke's experience. Clin Oncol. 2004;16(4):255-60.

6. Maddock-Jennings W, Wilkinson JM, Shillington D. Novel approaches to radiation-induced skin reactions: A literature review. Complement Ther Clin Pract. 2005;11(4):224-31.

7. Grootheest KV, DeJong-Van BLD. Patients' role in reporting adverse drug reactions. Expert Opin Drug Saf. 2004;3(4):363-8

8. Liekweg A, Westfeld M, Jaehde U. From oncology pharmacy to pharmaceutical care: New contribution to multidisciplinary cancer care. Support Care Cancer. 2004;12(2):73-9.

9. Aranda S, Jefford M, Yates P, Gough K, Seymour J, Francis P, et al. Impact of a novel nurseled prechemotherapy education intervention (ChemoEd) on patient distress, symptom burden and treatment-related information and support needs: results from arandomised, controlled trial. Ann Oncol. 2012;23(1):22231.

10. Carelle N, Piotto E, Bellanger A, Germanaud J, Thuillier A, Khayat D. Changing patient perceptions of the side effects of cancer chemotherapy. Cancer. 2002;95(1):155-63

11. Kayl AE, Meyers CA. Side-effects of chemotherapy and quality of life in ovarian and breast cancer patients. Curr Opin Obstet Gynecol. 2006;18(1):24-8

12. Cosentino M, Leoni $O$, Oria $C$, Michielotto D, Massimo E, Lecchini S, et al. Hospital-based survey of doctors' attitudes to adverse drug reactions and perception of drug-related risk for adverse reaction occurrence. Pharmacoepidemio Drug Saf. 1999;8(Suppl 1):S27-35.

13. Makowsky MJ, Koshman SL, Midodzi WK, Tsuyuki RT. Capturing outcomes of clinical activities performed by a rounding pharmacist practicing in a team environment: The COLLABORATE study [NCT00351676]. Med Care. 2009:47(6):642-50

14. Radiation Therapy Basics-American Cancer Society. How Radiation Therapy Can Affect Different Parts of the Body. [Updated 2014 December 10; cited 2016 November 20]. Available from https://www.cancer.org/treatment/treatments-andside-effects/treatment-types/radiation/effects-on-different-parts-of-body.html.

15. Berkey FJ. Managing the Adverse Effects of Radiation Therapy. Am Fam Physician. 2010;82(4):381-8

16. Feyer PCH, Maranzano E, Molassiotis A, Clark-Snow RA, Roila F, Warr D, et al. Radiotherapy-induced nausea and vomiting (RINV): antiemetic guidelines. Support Care Cancer. 2005;13(2):122-8.

17. Nicolini A, Ferrari P, Masoni MC, Fini M, Pagani S, Giampietro O, et al. Malnutrition, anorexia and cachexia in cancer patients: A mini-review on pathogenesis and treatment. Biomed Pharmacother. 2013;67(8):807-17.

18. Andreyev J. Gastrointestinal symptoms after pelvic radiotherapy: A new understanding to improve management of symptomatic patients. Lancet Oncol. 2007;8(11):1007-17.

19. Feyer P, Jahn F, Jordan K. Radiation induced nausea and vomiting. Eur J Pharmacol. 2014;722:165-71.

20. Horiot JC, Aapro M. Treatment implications for radiation-induced nausea and vomiting in specific patient groups. Eur J Cancer. 2004;40(7):979-87.

21. Hill A, Kiss N, Hodgson B, Crowe TC, Walsh AD. Associations between nutritional status, weight loss, radiotherapy treatment toxicity and treatment outcomes in gastrointestinal cancer patients. Clin Nutr. 2011;30(1):92-8.

22. Dewys WD, Begg C, Lavin P, Band PR, Bennett JM, Bertino JR, et al. Prognostic effect of weight loss prior to chemotherapy in cancer patients. Am J Med. 1980;69(4):491-7.

23. Robinson G, Goldstein M, Levine G. Impact of nutritional Status on DRG length of stay. J Parenter Enteral Nutr. 1987;11(1):49-51.

24. Ottery FD. Supportive nutrition to prevent cachexia and improve quality of life. Semin Oncol. 1995;22:98-111.

25. Li G, Jiang X, Qiu B, Shen LJ, Chen C, Xia YF. Vicious circle of acute radiation toxicities and weight loss predicts poor prognosis for nasopharyngeal carcinoma patients receiving intensity modulated radiotherapy. J Cancer. 2017:8(5):832-8.

26. Odelli C, Burgess D, Bateman L, Hughes A, Ackland S, Gillies J, et al. Nutrition Support Improves Patient Outcomes, Treatment Tolerance and Admission Characteristics in Oesophageal Cancer. Clin Oncol. 2005;17(8):639-45.

27. Epstein JB, Thariat J, Bensadoun RJ, Barasch A, Murphy BA, Kolnick L, et al. Oral complications of cancer and cancer therapy: from cancer treatment to sur vivorship. CA Cancer J Clin. 2012;62(6):400-22.

28. Herrstedt J. Prevention and management of mucositis in patients with cancer. Int J Antimicrob Agents. 2000;16(2):161-3.

29. Sonis ST, Elting LS, Keefe D, Peterson DE, Schubert M, Hauer-Jensen M, et al Perspectives on cancer therapy induced mucosal injury: Pathogenesis, measurement, epidemiology and consequences for patients. Cancer. 2004;100(9 suppl):1995-2025

30. Rosenthal DI, Trotti A. Strategies for Managing Radiation-Induced Mucositis in Head and Neck Cancer. Semin Radiat Oncol. 2009;19(1):29-34.

31. Eifel PJ, Jhingran A, Bodurka DC, Levenback $C$, Thames $H$. Correlation of smoking history and other patient characteristics with major complications of pelvic radiation therapy for cervical cancer. J Clin Oncol. 2002;20(17):3651-7.

32. Salvo N, Barnes E, Draanen JV, Stacey E, Mitera G, Breen D, et al. Prophylaxis and management of acute radiation-induced skin reactions: A systematic review of the literature. Curr Oncol. 2010;17(4):94-112.

33. Traeger L, Greer JA, Fernandez-Robles C, Temel JS, Pirl WF. Evidence-based treatment of anxiety in patients with cancer. J Clin Oncol. 2012;30(11):1197-205.

34. Al-taie A, Köseoğlu A. Incidence of Early Related-Complications of Port-A Catheter and Impact of Clinical Pharmacist Participation and Counselling Outcomes. J Young Pharm. 2018;10(2):218-21.

35. Thao KH, James AT. Assessment of Patients' Knowledge and Management of Chemotherapy Related Adverse Effects. J Hematol Oncol Pharm. 2014;4(4):122-7.

36. Izzettin FV, Al-taie A, Sancar M, Aliustaoğlu M. Influence of Pharmacist Recommendations for Chemotherapy-Related Problems in Diabetic Cancer Patients. Marmara Pharmaceutical Journal. 2017;21(3):603-11.

37. Krzyzanowska MK, Treacy J, Maloney B, Lavino A, Jacobson JO. Development of a patient registry to evaluate hospital admissions related to chemotherapy toxicity in a community cancer center. J Oncol Pract. 2005;1(1):15-9. 